\title{
HUMAN COMPUTER INTERACTION (HCI): HANDS FREE COMPUTER INTERACTION USING EYE MOVEMENT
}

\begin{tabular}{|l|l|}
\hline \multicolumn{1}{|c|}{ Sachin S } & Dr. Chetana Hegde \\
RNSIT (MCA), Bangalore & Associate Professor \\
sachin301190@gmail.com & $\begin{array}{l}\text { Dept. of MCA, RNSIT } \\
\text { chetanahegde@ieee.org }\end{array}$ \\
\hline
\end{tabular}

\begin{abstract}
This paper discusses about the human factors and technical considerations that uses eye movements as an interaction technique. An additional mode of communication between user and computer as an interface would be of great use. This technique of interaction is incorporated in a natural and unobtrusive way. The barrier to exploit this medium is because of the study of interaction techniques. The main aim is to communicate spatial information which is based on human eye movement, which is done without distracting the main reading task.
\end{abstract}

Key words- Non command based interface, Additional user input, Multi-Modal interaction.

\section{INTRODUCTION}

Now a days, user-computer interaction is one sided, with the bandwidth from the computer to the user far than that from user to computer.

The eye retina is not uniform, where one small portion near its center has many densely-packed receptors and thus permits sharp vision, and the remaining part of the retina permits only much blurrier vision. Anything outside this area is seen with "peripheral vision". This human computer interactions are like two powerful information processors (human and computer) attempting to communicate with each other via a narrow-bandwidth.

In addition, eye movement-based interaction techniques provide an interesting example of a new, non- command style of interaction. Some of the qualities are like the user does not issue specific commands, instead, the computer observes the user and provides appropriate responses.

\subsection{HUMAN-COMPUTER INTERACTION (HCI):}

Most of the sophisticated machines are of no use unless they are properly used by people. This argument simply presents the main terms that should be considered in the design of HCI: functionality and usability.
Functionality of a system is the set of actions or services provided to its users. However, the value of functionality is visible when it is available for efficient usage.

Usability of a system with a certain functionality is the range at which the system is used efficiently and adequately to accomplish achieve goals for users.

The system effectiveness is achieved only when there is a proper balance between the functionality and usability of a system.

Determining what makes a HCI design good is that it is mostly subjective dependent and context dependent. For example, the designing tool for an aircraft part should provide high precisions in design of the parts whereas, some other graphics editing software may not require such a precision. Currently available technology could also affect as to how different types of HCI are designed for the same purpose. One such example is using GUI, menus, virtual reality or commands to access its functionalities for any given computer.

\section{LITERATURE SURVEY:}

The existing interfaces vary in the degree of complexity and also in the financial and economical aspect of the machine in market. On the other hand, a simple website also may be limited in the functionality and it should be complex to attract and keep the customers.

The focus here is on the advances in physical aspect of interaction and also to show how different methods of interaction can be combined (Multi-Modal Interaction) and also how each method is improved in performance (Intelligent Interaction) for providing a better and easier interface for the user.

There is a considerable amount of research using eye tracking, but it has concentrated on eye movement data as a tool for studying motor and cognitive processes. In the design of this HCI, the level of activity that involves the user with a machine should be considered effectively. There are three different levels in the user activity: physical [1], cognitive [2] and affective [3]. 
The physical aspect is what which determines the mechanics of interaction between human and computer while the cognitive aspect is what which deals with the way that the users can understand the system and also interact with it.

The affective aspect is a more recent issue which tries not only to make the interaction a pleasurable experience for the user but also to affect the user in a way that makes user to continue to use the machine by changing attitudes and emotions towards the user.

\section{METHODOLOGY:}

The existing interfaces differ in the degree of complexity both because of degree of functionality/usability and the financial and economical aspect of the machine in market.

\subsection{METHODS FOR MEASURING EYE MOVEMENTS}

First, our goal is to measure visual line of gaze, which is the absolute position in space at which the human's/user's eyes is pointed.

The principal method for moving a different portion of the visual scene is a sudden and rapid motion called a saccade. Angle (15-20 degrees being most typical). Saccades take approximately 30-120 milliseconds and traverse a range between 1 and 40 degrees of visual.

Figure 1 shows the computer user's eye movements over approximately 30 seconds, while on normal work.

More practical methods use remote imaging of a visible feature located on the eyeball, such as the boundary between the sclera and iris, the outline of the pupil. Typically, a saccade is followed by a $200-600 \mathrm{~ms}$ period of relative stability, called a fixation, during which the eye does not remain still.

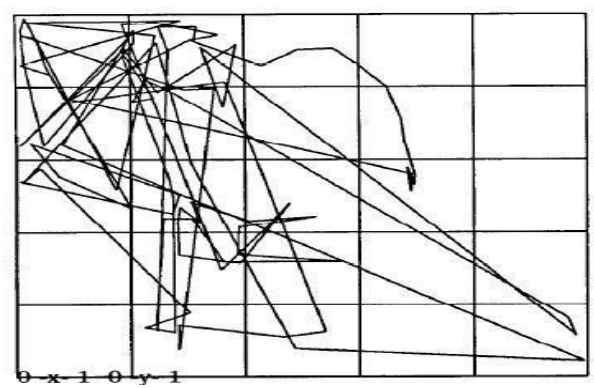

Figure 1 Eye movement of user on computer screen.

\subsection{CHARACTERSTICS OF EYE MOVEMENTS}

If we need to see an object with clarity, it is necessary to move the eyeball, so the object appears on the fovea, which is small area at the center of the retina.

During a fixation, however, the eye still makes small, jittery motions, generally covering less than one degree. Smooth eye motions, less sudden than saccades, occur only in response to a moving object in the visual field.

The eyes are rarely entirely still. They move during a fixation, and seldom remain in one fixation for long.

\section{HCI SYSTEM ARCHITECTURE}

One of the most important factor of the HCI design is its configuration. Architecture of the HCI system shows what are the inputs and outputs and how they work together.

1. Unimodal HCL Systems

2. Multimodal HCL Systems

\subsection{UNIMODAL HCL SYSTEMS}

A system that is based on only one modality is called unimodal. Each of the different independent single channels is called a modality.

Based on the nature of different modalities, they can be divided into three categories:

1. Visual-Based

2. Audio-Based

3. Sensor-Based

\subsubsection{VISUAL-BASED HCI}

The visual based human computer interaction is probably the most widespread area in HCI research. Some of the main research areas in this section are as follow:

- Facial Expression Analysis

- Body Movement Tracking (Large-scale)

- Gesture Recognition

- Gaze Detection (Eyes Movement Tracking)

Facial expression analysis generally deals with recognition of emotions visually. The main focus of this area is body movement tracking and gesture recognition for direct interaction of human and computer in a command and action scenario.

Gaze detection is mostly an indirect form of interaction between user and machine which is mostly used for better understanding of user's attention, intent or focus in contextsensitive situations. 


\subsubsection{AUDIO-BASED HCI}

The audio based interaction between a computer and a human is another important area of HCI systems. This area deals with information acquired by different audio signals. While the nature of audio signals may not be as variable as visual signals but the information gathered from audio signals can be more trustable, helpful, and is some cases unique providers of information. Research areas in this section can be divided to the following parts:

- Speech Recognition

- Speaker Recognition

- Auditory Emotion Analysis

- Human-Made Noise/Sign Detections (Gasp, Laugh, Cry, etc.)

- Musical Interaction

\subsubsection{SENSOR-BASED HCI}

This section is a combination of variety of areas with a wide range of applications. The commonality of these different areas is that at least one physical sensor is used between user and machine to provide the interaction. These sensors as shown below can be very primitive or very sophisticated.

1. Pen-Based Interaction

2. Mouse \& Keyboard

3. Joysticks

4. Motion Tracking Sensors and Digitizers

5. Pressure Sensors

\subsection{MULTIMODAL HCL SYSTEMS}

Multimodal is the combination of multiple modalities. In MM HCI systems, these modalities are the ways that the system responds to the inputs, i.e. communication channels. Multimodal interface acts as a facilitator of human-computer interaction via two or more modes of input that go beyond the traditional keyboard and mouse.

In spite of all progress made in MMHCI, in most of existing multimodal systems, the modalities are still treated separately and only at the end, results of different modalities are combined together.

An interesting aspect of multimodality is the collaboration of different modalities to assist the recognitions. For example, lip movement tracking (visual- based) can help speech recognition methods (audio-based) and speech recognition methods (audiobased) can assist command acquisition in gesture recognition (visual-based). The next section shows some of application of intelligent multimodal systems.

\section{APPLICATIONS}

Multimodal interfaces can offer a number of advantages over traditional interfaces. For one thing, they can offer a more natural and user-friendly experience. In a noisy setting, one may provide input through handwriting rather than speech. Few other examples of applications of multimodal systems are listed below:

- Smart Video Conferencing

- Intelligent Homes/Offices

- Driver Monitoring

- Intelligent Games

- E-Commerce

- Helping People with Disabilities

\section{CONCLUSIONS}

An eye tracker as an input device is far from "perfect" in the sense that a mouse or keyboard is, and that is caused both by the limitations of current equipment and, more importantly, by the nature of human eye movements.

\section{REFERENCES}

[1] A. Chapanis, Man Machine Engineering, Wadsworth, Belmont (1965).

[2] D. Norman, "Cognitive Engineering", in D. Norman and S. Draper (eds), User Centered Design: New Perspective on HumanComputer Interaction, Lawrence Erlbaum, Hillsdale (1986).

[3] R.W. Picard, Affective Computing, MIT Press, Cambridge (1997). 